\title{
Online Distributed Network Traffic Signal Control using the Cell Transmission Model
}

\author{
Stelios Timotheou, Christos G. Panayiotou and Marios M. Polycarpou \\ Department of Electrical and Computer Engineering, University of Cyprus \\ \{timotheou.stelios, christosp, mpolycar\}@ucy.ac.cy
}

\begin{abstract}
This paper considers the solution of the adaptive network traffic signal control problem under a fully distributed architecture. To achieve a system-wide optimal solution, the problem is modeled as a large-scale mixed integer linear program with the traffic dynamics being captured by the cell transmission model. To achieve an online distributed solution to the considered problem, the loosely connected structure of transportation networks is exploited to decompose the problem in both space and time. The proposed solution methodology involves two main phases. In the first phase, binary decision variables are relaxed and the resulting linear program is distributedly solved via the alternating direction method of multipliers. The second phase involves distributed rounding of the obtained relaxed solution. Simulation results demonstrate the effectiveness of the proposed approach in providing close to optimal, online solutions.
\end{abstract}

Keywords: traffic signal control, alternating direction method of multipliers, online, distributed, CTM.

\section{INTRODUCTION}

Traffic signal control can bring substantial reduction to traffic congestion leading to improved conditions both for the drivers (better travel times and convenience) and the environment (reduced air pollution and energy consumption). Furthermore, recent advancements in electronics, sensing, and ICT (information and communication technology) allow the real-time collection and processing of traffic data, as well as the deployment of intelligent controllers for the efficient operation of a transportation system. However, controlling the traffic signals at the network level constitutes a significant challenge due to the large-scale, uncertain and dynamic nature of the problem. Hence, several solution approaches have been proposed to provide efficient solutions to the network traffic signal control problem.

Some techniques consider the control of isolated intersections [1], while the majority of techniques examine the network traffic signal control (NTSC) problem where the goal is to optimize the performance of the entire network consisting of numerous intersections. Fixed or pre-timed signal control strategies optimize offline the signal timing plans based on historical data so that fixed signal programs are applied for different periods of the day. Fixed-time NTSC methods either attempt to adjust the offset between adjacent intersections so as to maximize progression along multiple corridors, e.g. in MULTIBAND [2], or optimize split plans and cycle according to some measure of effectiveness (MOE) that combines different traffic metrics

This work is partially funded by the European Research Council Advanced Grant FAULT-ADAPTIVE (ERC-2011-AdG-291508). such as delay, minimum number of stops and throughput, e.g. TRANSYT [3]. Cell transmission model(CTM)-based optimization approaches usually rely on the development of mixed integer linear programming (MILP) formulations [4] which are usually NP-hard; hence, greedy heuristics [5] are often employed to achieve close to optimal and timely solutions. Pre-timed signal control strategies can perform fairly well during peak traffic periods, but their performance deteriorates during off-peak periods or when unexpected events create different traffic conditions than anticipated.

To account for stochastic variations of traffic flows, several online adaptive traffic signal control (ATSC) systems have been developed. ATSC approaches collect information from different sources on-demand, and use them to adaptively optimize traffic signal plan parameters such as splits, offsets and cycle, (e.g. SCOOT, [6], RHODES [7] ) or select the best from a library of pre-calculated signal plans (e.g. SCATS [8]). Such systems usually rely on centralized architectures where one computing unit decides for all intersections (e.g. SCOOT, SCATS) or hierarchical architectures where the decision is partly centralized and partly decentralized (e.g. RHODES).

Fully distributed online algorithms dynamically adjust the split plans of multiple intersections based on the network state. Due to the complex and large-scale nature of the problem these algorithms employ simplistic system models that lead to low-complexity but inaccurate techniques. Such techniques usually draw inspiration from artificial intelligence, such as multi-agent reinforcement learning (RL) [9], and control theory, such as back-pressure routing [10].

In this paper a distributed cooperative online algorithm for the NTSC problem based on CTM traffic dynamics is proposed. Distributed cooperative optimization is achieved by iteratively solving one intersection subproblems and exchanging information between adjacent intersection controllers. Online optimization is accomplished by adapting traffic signal plans every one small time-window (e.g. of $10 \mathrm{mins}$ ), by proactively attaining traffic plans every one time-window and applying them for the next one. In this way, our approach departs from the traditional view of considering optimization techniques for the solution of CTM-based NTSC problems, only for offline centralised systems. The solution approach for the MILP CTM-based NTSC problem is comprised of two phases. In the first phase, the relaxed problem is solved via the alternating direction method of multipliers (ADMM) [11]; the resulting solution is utilized in the second phase to obtain a solution to the original problem via distributed rounding. 
The remainder of this paper is organized as follows. Section II-A, outlines CTM, while Section II-B introduces ADMM. Section III presents the centralized formulation of the NTSC problem. Section IV explains the two phases of the distributed solution approach. Section V, examines the performance of the proposed approach, while Section VI summarizes the paper.

\section{PRELIMINARIES}

\section{A. Cell Transmission Model}

The Cell Transmission Model (CTM) [12] is a discrete analog in both space and time of the well-known first-order Lighthill-Whitham-Richards (LWR) continuum flow model. In CTM, each road segment is divided into homogeneous sections called cells, while time is partitioned in a way that one vehicle takes one time-unit to travel through one cell at free-flow speed (the speed of vehicles when density is zero). Assuming a piecewise-linear flow-density fundamental diagram, CTM equations are given by:

$$
\begin{aligned}
y_{c, t} & =\min \left(n_{c, t}, q_{c, t}, W_{c+1, t} \bar{n}_{c+1, t}\right) \\
\bar{y}_{c+1, t} & =y_{c, t} \\
n_{c, t+1} & =n_{c, t}+\bar{y}_{c, t}-y_{c, t}
\end{aligned}
$$

In the above equations, $\bar{y}_{c, t}, y_{c, t}, n_{c, t}$ and $\bar{n}_{c+1, t}$ represent the number of vehicles entering cell $c$, leaving cell $c$, are inside cell $c$ and needed to reach maximum occupancy of cell $c$ respectively, at time $[t, t+1)$. Parameter $q_{c, t}$ is the capacity of cell $c$ which denotes the maximum number of vehicles that can flow out of cell $c$ and enter subsequent cells. The values of $\bar{y}_{c, t}, \bar{n}_{c, t}$ and $q_{c, t}$ depend on the type and characteristics of the cell (e.g. origin, destination, intersection cell). Important characteristics of cells include the number of vehicles entering cell $c$ from the outside of the network $\left(D_{c, t}\right)$, the maximum number of vehicles that can flow through $\left(Q_{c, t}\right)$ or reside into cell $c\left(N_{c, t}\right)$ at time $[t, t+1)$, respectively. $W_{c, t}$ is the ratio between the shock-wave propagation speed and the free-flow speed and indicates how fast a vehicle queue is formed. In homogeneous networks, it is true that quantities $Q_{c, t}=Q, N_{c, t}=N$ and $W_{c, t}=W$ are constant for all cells. Eq. (1) indicates that the number of vehicle leaving cell $c$ is limited either by the number of vehicles in the cell, the capacity of the cell for outflow vehicles, the capacity of the successor cell for inflow vehicles and the space left in the successor cell when a queue is forming. Eqs. (2) - (3) ensure flow conservation at cell $c$. In section III, CTM is utilized to derive a MILP formulation for the NTSC problem.

\section{B. Alternating Direction Method of Multipliers}

ADMM is a powerful method for solving mathematical optimization problems of the form

$$
\begin{array}{cc}
\min & f(\mathbf{x})+g(\mathbf{z}) \\
\text { s.t. } & \mathbf{A x}+\mathbf{B} \mathbf{z}=\mathbf{d} \\
& \mathbf{x} \in \mathcal{C}_{x}, \mathbf{z} \in \mathcal{C}_{z}
\end{array}
$$

where $\mathbf{x} \in \mathcal{C}_{x} \subseteq \mathbb{R}^{M_{x} \times 1}, \mathbf{z} \in \mathcal{C}_{z} \subseteq \mathbb{R}^{M_{z} \times 1}, \mathbf{A} \in \mathbb{R}^{M_{d} \times M_{x}}$, $\mathbf{B} \in \mathbb{R}^{M_{d} \times M_{z}}$ and $\mathbf{d} \in \mathbb{R}^{M_{d} \times 1}, f(\mathbf{x})$ and $g(\mathbf{z})$ are convex functions and $\mathcal{C}_{x}, \mathcal{C}_{z}$ are closed convex sets. ADMM is capable of providing fast and often distributed solutions to convex problems through appropriate decomposition of the considered problem into simpler ones [11]. For the solution of problem (4), ADMM uses the scaled augmented Lagrangian form, $\mathcal{L}_{\rho}(\mathbf{x}, \mathbf{z}, \mathbf{u})$ :

$$
\begin{aligned}
\mathcal{L}_{\rho}(\mathbf{x}, \mathbf{z}, \mathbf{u})= & f(\mathbf{x})+g(\mathbf{z})+h_{\mathcal{C}_{x}}(\mathbf{x})+h_{\mathcal{C}_{z}}(\mathbf{z})+ \\
& (\rho / 2)\|\mathbf{A} \mathbf{x}+\mathbf{B z}-\mathbf{d}+\mathbf{u}\|_{2}^{2}
\end{aligned}
$$

where $\mathbf{u}=\boldsymbol{\omega} / \rho$ are the scaled dual variables, $\boldsymbol{\omega} \in \mathbb{R}^{M_{d} \times 1}$ are the dual variables or Lagrange multipliers and $\rho \in \mathbb{R}$ is a penalty constant, while $h_{\mathcal{C}_{x}}(\mathbf{x})$ and $h_{\mathcal{C}_{z}}(\mathbf{z})$ are indicator functions which are equal to zero if $\mathbf{x} \in \mathcal{C}_{x}$ and $\mathbf{z} \in \mathcal{C}_{z}$, and $+\infty$ otherwise. Starting from initial values $\mathbf{z}^{0}$ and $\mathbf{u}^{0}$ ADMM iteratively minimizes $\mathcal{L}_{\rho}(\mathbf{x}, \mathbf{z}, \mathbf{u})$ with respect to $\mathbf{x}$ and $\mathbf{z}$ followed by an update of the scaled dual variables in three consecutive steps:

$$
\begin{aligned}
& \text { Step 1: } \mathbf{x}^{k+1}=\arg \min _{\mathbf{x}} \mathcal{L}_{\rho}\left(\mathbf{x}, \mathbf{z}^{k}, \mathbf{u}^{k}\right) \\
& \text { Step 2: } \mathbf{z}^{k+1}=\arg \min _{\mathbf{z}} \mathcal{L}_{\rho}\left(\mathbf{x}^{k+1}, \mathbf{z}, \mathbf{u}^{k}\right) \\
& \text { Step 3: } \mathbf{u}^{k+1}=\mathbf{A} \mathbf{x}^{k+1}+\mathbf{B} \mathbf{z}^{k+1}-\mathbf{d}+\mathbf{u}^{k}
\end{aligned}
$$

The procedure continues until a stopping criterion is satisfied. Contrary to other decomposition methods that impose strong convergence conditions, ADMM enjoys the superior convergence properties of the method of multipliers with mild technical convergence conditions.

\section{Centralized Problem Formulation}

We consider the problem of jointly optimizing the traffic signal plans of a transportation network with multiple intersections over a time-horizon $T_{h}$. Optimization is performed for the mean/total vehicle delay but other measures of interest such as stoppage time and throughput can also be employed. Traffic dynamics are incorporated into the optimization problem through CTM described in section II-A. To simplify the problem, we separate $T_{h}$ into smaller time-windows, $T_{w}$, and optimize the problem sequentially over those periods. The optimization period considered for each subproblem $T_{o}$, is larger than $T_{w}$ to avoid the effects of short-term planning, while generated traffic can be considered for $T_{f} \geq T_{w}$ time units. In fact, when online decision making is sought, traffic signal plans must be updated every $T_{w}$ time units, using only the solution corresponding to the first $T_{w}$ time units.

We consider a grid transportation network with two way streets and $N_{I}$ intersections, as shown in Fig. 1. To model the traffic dynamics four types of CTM cell sets are utilized: ordinary $(\mathcal{E})$, origin $(\mathcal{O})$, destination $(\mathcal{D})$ and intersection $(\mathcal{I})$, with $\mathcal{A}=\mathcal{E} \cup \mathcal{O} \cup \mathcal{D} \cup \mathcal{I}$. We assume that traffic lights have two phases so that cells of intersection $i \in \mathcal{R}=\left\{1, \ldots, N_{I}\right\}$ receive a green light either at phase 1 or phase 2 (sets $\mathcal{I}_{1, i}$ and $\mathcal{I}_{2, i}$, respectively).

Assuming that decision variables $w_{i, t}$, indicate whether phase $1\left(w_{i, t}=1\right)$ or phase $2\left(w_{i, t}=0\right)$ has the right-ofway at intersection $i \in \mathcal{R}$ at time $t \in \mathcal{T}=\left\{1, \ldots, T_{o}\right\}$, the 
NTSC problem can be formulated as:

$$
\begin{aligned}
& \mathcal{N} \mathcal{T S C}: \min \sum_{c \in \mathcal{A}} \sum_{t \in \mathcal{T}} n_{c, t}-\sum_{c \in \mathcal{A}} \sum_{t \in \mathcal{T}} y_{c, t+1}, \\
& \text { s.t. } 0 \leq y_{c, t} \leq q_{c, t}, y_{c, t} \leq n_{c, t}, c \in \mathcal{A}, t \in \mathcal{T} \text {, } \\
& y_{c, t} \leq W_{c+1, t} \bar{n}_{c+1, t}, c \in \mathcal{A}, t \in \mathcal{T}, \\
& n_{c, t+1}=n_{c, t}+\bar{y}_{c, t}-y_{c, t}, c \in \mathcal{A}, t \in \mathcal{T} \text {, } \\
& q_{c, t}= \begin{cases}Q_{c, t}, & c \in \mathcal{E} \cup \mathcal{O}, t \in \mathcal{T} \\
w_{i, t} Q_{c, t}, & i \in \mathcal{R}, c \in \mathcal{I}_{1, i}, t \in \mathcal{T} \\
\left(1-w_{i, t}\right) Q_{c, t}, & i \in \mathcal{R}, c \in \mathcal{I}_{2, i}, t \in \mathcal{T} \\
\infty, & c \in \mathcal{D}, t \in \mathcal{T}\end{cases} \\
& \bar{n}_{c+1, t}= \begin{cases}N_{c+1, t}-n_{c+1, t}, & c \in \mathcal{A} \backslash \mathcal{D}, t \in \mathcal{T} \\
\infty, & \text { otherwise }\end{cases} \\
& \bar{y}_{c, t}= \begin{cases}y_{c-1, t}, & c \in \mathcal{A} \backslash \mathcal{O}, t \in \mathcal{T} \\
D_{c, t}, & \text { otherwise }\end{cases} \\
& w_{i, t}-w_{i, t-1} \leq u_{i, t} \leq w_{i, t}+w_{i, t-1}, \quad i \in \mathcal{R}, t \in \mathcal{T}, \\
& -w_{i, t}+w_{i, t-1} \leq u_{i, t} \leq 2-w_{i, t}-w_{i, t-1}, \quad i \in \mathcal{R}, t \in \mathcal{T} \\
& \sum_{\tau=t}^{t+G_{\min }} u_{i, \tau} \leq 1, i \in \mathcal{R}, t=1, \ldots, T_{o}-G_{\text {min }}, \\
& \sum_{\tau=t}^{t+G_{\max }-1} w_{i, \tau} \leq G_{\max }, i \in \mathcal{R}, t=1, \ldots, T-G_{\max }+1, \\
& t+G_{\max }-1 \\
& \sum_{\tau=t}^{G_{\max }} w_{i, \tau} \geq 1, i \in \mathcal{R}, t=1, \ldots, T-G_{\max }+1, \\
& w_{i, t}=w_{i, t}^{i n i t}, i \in \mathcal{R}, t=-G_{\max }+1, \ldots, 0, \\
& n_{c, 1}=n_{c}^{i n i t}, c \in \mathcal{A} \text {, } \\
& w_{i, t} \in\{0,1\}, 0 \leq u_{i, t} \leq 1, i \in \mathcal{R}, t \in \mathcal{T} .
\end{aligned}
$$

In the above formulation, (5a) minimizes the total delay, as $\left(n_{c, t}-y_{c, t+1}\right)$ denotes the delay experienced by vehicles in cell $c$ at time $t$ in time units; minimizing the total delay is equivalent to minimizing the total travel time (TTT), because in CTM vehicles either travel at free-flow speed or are motionless due to congestion. Constraints $(5 b)-(5 d)$, represent the fundamental CTM dynamics, while (5e)-(5g), define cell parameters $q_{c, t}, \bar{n}_{c, t}$ and $\bar{y}_{c, t}$, respectively for different types of cells. Inequalities (5h)-(5i) are equivalent to $u_{i, t}=\left|w_{i, t}-w_{i, t-1}\right|$, when $w_{i, t} \in\{0,1\}$; hence $u_{i, t}$ indicates whether a signal change has occurred between $t-1$ and $t$ (green-to-red or red-to-green). Inequalities (5j)-(5l) ensure that the minimum and maximum duration of each phase is $G_{\min }$ and $G_{\max }$, respectively, while (5m)-(5n) are initial conditions that need to be respected regarding the state of the network and the traffic signals. Without loss of generality, we will assume for the rest of the paper that the network is homogeneous. By relaxing the main decision variables $w_{i, t}$ to take continuous values, i.e. $w_{i, t} \in[0,1]$ we arrive at the relaxed formulation, $\mathcal{R N} \mathcal{T S C}$. More details regarding the centralised formulation can be found in [13].

\section{IV. $\mathcal{N} \mathcal{T} \mathcal{S C}$ DISTRIBUTED FORMULATION AND SOLUTION}

In this paper we consider the solution of the $\mathcal{N T S C}$ problem assuming a fully distributed architecture with each signaled intersection equipped with an intelligent controller that has sensing, communication, computation and control capabilities. A distributed solution to $\mathcal{N} \mathcal{T S C}$ is obtained by performing two consecutive phases. In phase 1 , the relaxed problem, $\mathcal{R N} \mathcal{N} \mathcal{S C}$, is solved distributedly via $\mathrm{ADMM}$, by appropriate reformulation of the problem, so as to avoid the

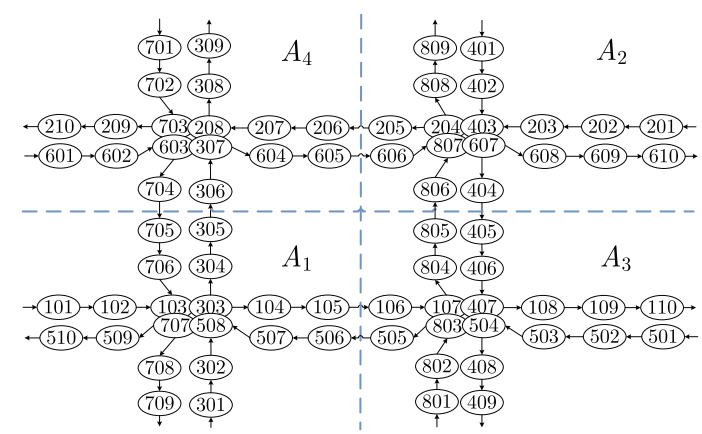

Fig. 1. CTM for a 2-by-2 intersection topology. The dashed lines indicate the spatial decomposition of the network into four areas, each one controlled by the corresponding intersection controller.

exponential complexity of $\mathcal{N} \mathcal{T S C}$. In phase 2 , a distributed rounding scheme is used to obtain a close to optimal solution to $\mathcal{N} \mathcal{T S C}$ by rounding-off fractional values of decision variables obtained from the solution of $\mathcal{R N} \mathcal{T S C}$ in phase 1 .

\section{A. Spatial Decomposition}

The distributed solution of the NTSC problem is motivated by the special structure of transportation networks that allow appropriate spatial decomposition, as explained through the example topology in Fig. 1, which depicts the CTM of a $2 \times 2$ grid topology with four intersections and two-way arterials. In the figure, the dashed lines indicate a possible spatial decomposition of the network which partitions the topology into four areas $A_{i}, i=1, \ldots, 4$ so that each area is associated to exactly one intersection. Note that under such a decomposition, interactions between adjacent intersections only take place through boundary cells, while if the optimal incoming boundary flows are known for each intersection, the optimal solution to the global problem can be obtained by independently solving single-intersection subproblems.

This can be understood by observing the characteristic CTM equations $(5 b)-(5 g)$, from which it is clear that the computation of variables $\left(y_{c, t}, n_{c, t+1}\right)$ for ordinary cells only depends on variables associated with cells $c-1, c$ and $c+1$. This important observation, implies that the cell states can evolve independently for each area, apart from boundary cells which require information about variables that belong to predecessor or successor cells outside the particular area.

This discussion gives rise to two different types of boundary cells: (a) input and (b) output. An input boundary cell $c \in \mathcal{B}_{i}^{I}$ is any boundary cell of area $A_{i}$ that receives inflow traffic from a cell of a neighboring area (e.g. cell 705). Similarly, an output boundary cell $c \in \mathcal{B}_{i}^{O}$ is any boundary cell of area $A_{i}$ that sends outflow traffic to a cell of a neighboring area (e.g. cell 105). We also define the extended input and output boundary cell sets of area $A_{i}$, $\mathcal{B}_{i}^{I E}$ and $\mathcal{B}_{i}^{O E}$ respectively, which include all input/output cells associated with area $A_{i}$ and input/output cells that are directly adjacent to $A_{i}$, as well as the set of all boundary cells of area $A_{i}, \mathcal{B}_{i}=\mathcal{B}_{i}^{I} \cup \mathcal{B}_{i}^{O}$ and the corresponding extended set $\mathcal{B}_{i}^{E}=\mathcal{B}_{i}^{I E} \cup \mathcal{B}_{i}^{O E}$. These definitions facilitate the distributed solution of the NTSC problem discussed next. 


\section{B. Phase 1: Distributed Solution of $\mathcal{R N} \mathcal{N} \mathcal{S C}$}

To derive a distributed formulation for $\mathcal{R N} \mathcal{T S C}$, we need to convert the problem into an iterative procedure composed of single-intersection subproblems, so that each intersection controller (IC) only solves problems involving local variables. Towards this direction, we rewrite the centralised $\mathcal{R N} \mathcal{T S C}$ problem into the following form that distinguishes decoupled constraints for each intersection (Eqs. (6b), (6c) and (6d)), from coupled constraints that involve variables from more than one intersections (Eqs. (6e) and (6f)):

$$
\begin{aligned}
\min & \sum_{i \in \mathcal{R}} \mathbf{f}_{i}^{T} \mathbf{x}_{i} \\
\text { s.t. } & \mathbf{A}_{i} \mathbf{x}_{i}=\mathbf{a}_{i}, i \in \mathcal{R}, \\
& \mathbf{B}_{i} \mathbf{x}_{i} \leq \mathbf{b}_{i}, i \in \mathcal{R}, \\
& \mathbf{x}_{i}^{l} \leq \mathbf{x}_{i} \leq \mathbf{x}_{i}^{u}, i \in \mathcal{R}, \\
& y_{c, t}+s_{c, t}=W N-W n_{c+1, t}, c \in \mathcal{B}_{i}^{O}, t \in \mathcal{T}, i \in \mathcal{R}, \\
& n_{c, t+1}=n_{c, t}+y_{c-1, t}-y_{c, t}, c \in \mathcal{B}_{i}^{I}, t \in \mathcal{T}, i \in \mathcal{R},
\end{aligned}
$$

where $\mathbf{x}_{i}=\left[\mathbf{n}_{i}^{T}, \mathbf{y}_{i}^{T}, \mathbf{w}_{i}^{T}, \mathbf{u}_{i}^{T}, \mathbf{s}_{i}^{T}\right]^{T}$ denotes the vector of variables of area $A_{i}, \mathbf{n}_{i} \in \mathbb{R}^{\left|\mathcal{A}_{i}\right|\left(T_{o}+1\right) \times 1}, \mathbf{y}_{i} \in \mathbb{R}^{\left|\mathcal{A}_{i}\right| T_{o} \times 1}$, $\mathbf{w}_{i} \in \mathbb{R}^{T_{o} \times 1}, \mathbf{u}_{i} \in \mathbb{R}^{\left(T_{o}-1\right) \times 1}$ are vectorized versions of variables $n_{c, t}, y_{c, t}, w_{i, t}$ and $u_{i, t}, c \in \mathcal{A}_{i}, i \in \mathcal{R}, t \in \mathcal{T}$, $\mathbf{x}_{i}^{l}$ and $\mathbf{x}_{i}^{u}$ are the lower and upper bounds of the corresponding variables, while slack variables $\mathbf{s}_{i} \in \mathbb{R}^{\left|\mathcal{B}_{i}^{O}\right| T_{o} \times 1}$ are introduced to convert inequalities $(5 \mathrm{c})$ for output boundary cells of area $A_{i}$ into equalities. Note that area-related sets are denoted with the same symbol and a subscript index to indicate the area, e.g. $\mathcal{A}_{i}$ is the set of all cells in area $A_{i}$. Expression (6a) captures the linear objective function, where vector $\mathbf{f}_{i}$ denotes the cost associated with variables $\mathbf{x}_{i}$, while Eqs. (6b) and (6c) capture all decoupled equality and inequality constraints whose variables belong to the same area i.e. Eqs. (5b), (5e)-(5o), (5c) excluding output boundary cells, and (5d) excluding input boundary cells. The excluded constraints are represented by the coupled equalities (6e) and (6f) which involve variables from two different areas and hence cannot be handled directly by the same IC. Note that any initialization constraints have been included in the above formulation as bound constraints.

To be able to solve the problem in a distributed manner via the ADMM algorithm, we introduce auxiliary variables $\mathbf{z}$ and rewrite (6) into the equivalent form:

$$
\begin{aligned}
& \min \sum_{i \in \mathcal{R}} \mathbf{f}_{i}^{T} \mathbf{x}_{i} \\
& \text { s.t.Constraints (6b), (6c) and (6d), } \\
& \quad y_{c, t}+s_{c, t}=W N-W z_{c+1, t}^{n}, \quad c \in \mathcal{B}_{i}^{O}, t \in \mathcal{T}, i \in \mathcal{R}, \\
& n_{c, t+1}=n_{c, t}+z_{c-1, t}^{y}-y_{c, t}, \quad c \in \mathcal{B}_{i}^{I}, t \in \mathcal{T}, i \in \mathcal{R}, \\
& z_{c+1, t}^{n}=n_{c+1, t}, \quad c \in \mathcal{B}_{i}^{O}, t \in \mathcal{T}, i \in \mathcal{R}, \\
& z_{c-1, t}^{y}=y_{c-1, t}, \quad c \in \mathcal{B}_{i}^{I}, t \in \mathcal{T}, i \in \mathcal{R},
\end{aligned}
$$

For better clarity, variables $z_{c, t}^{n}$ correspond to variables $n_{c, t}$; the same applies to the other variable sets. Note also that (7e) and (7f) can be equivalently expressed as $z_{c, t}^{n}=n_{c, t}$, $c \in \mathcal{B}_{i}^{I}, t \in \mathcal{T}, i \in \mathcal{R}$, and $z_{c, t}^{y}=y_{c, t}, c \in \mathcal{B}_{i}^{O}, \quad t \in \mathcal{T}$, $i \in \mathcal{R}$, respectively.

The above formulation adheres to ADMM formulation (4) with $\mathbf{x}=\left[\mathbf{x}_{1}^{T}, \ldots, \mathbf{x}_{N_{I}}^{T}\right]^{T}$ and $\mathbf{z}=\left[\mathbf{z}_{1}^{T}, \ldots, \mathbf{z}_{N_{I}}^{T}\right]^{T}$, with $\mathbf{z}_{i}$ denoting the vector of elements $z_{c, t}^{n}, c \in \mathcal{B}_{i}^{O}, t \in \mathcal{T}$ and $z_{c, t}^{y}, c \in \mathcal{B}_{i}^{I}, t \in \mathcal{T}$. Convex set $\mathcal{C}_{z}$ is empty, while $\mathcal{C}_{x}=$ $\{\mathbf{x} \mid$ Constraints (6b), (6c) and (6d) $\}$. Constraints (7c)-(7f) are coupling constraints between $\mathrm{x}$ and $\mathrm{z}$, representing eq. (4b) of ADMM formulation.

According to the ADMM algorithm we rewrite the problem into the augmented lagrangian form to obtain:

$$
\begin{aligned}
& \min \mathcal{L}(\mathbf{x}, \mathbf{z}, \boldsymbol{\lambda}, \boldsymbol{\mu}, \boldsymbol{\nu})= \\
& \quad \sum_{i \in \mathcal{R}}\left(\mathbf{f}_{i}^{T} \mathbf{x}_{i}+h_{\mathcal{C}_{x_{i}}}\left(\mathbf{x}_{i}\right)+\frac{\rho}{2}\left\|\mathbf{x}_{i}-\mathbf{z}_{i}+\boldsymbol{\nu}_{i}\right\|_{2}^{2}\right. \\
& \quad+\frac{\rho}{2} \sum_{c \in \mathcal{B}_{i}^{O}} \sum_{t \in \mathcal{T}}\left(y_{c, t}+s_{c, t}+W z_{c+1, t}^{n}-W N+\lambda_{c, t}\right)^{2} \\
& \left.\quad+\frac{\rho}{2} \sum_{c \in \mathcal{B}_{i}^{I}} \sum_{t \in \mathcal{T}}\left(n_{c, t+1}-n_{c, t}+y_{c, t}-z_{c-1, t}^{y}+\mu_{c, t}\right)^{2}\right),
\end{aligned}
$$

where $\boldsymbol{\nu}, \boldsymbol{\lambda}$, and $\boldsymbol{\mu}$ are the scaled Lagrange multipliers of constraints (7e)-(7f), (7c), and (7d), respectively. Hence, ADMM consists of iteratively solving $\mathcal{L}(\mathbf{x}, \mathbf{z}, \boldsymbol{\lambda}, \boldsymbol{\mu}, \boldsymbol{\nu})$ over $\mathbf{x}$ (step 1), then over $\mathbf{z}$ (step 2), and then updating $\boldsymbol{\lambda}, \boldsymbol{\mu}, \boldsymbol{\nu}$ (step $3)$. After the $k$ th iteration of the ADMM algorithm, vectors $\mathbf{x}^{k}, \mathbf{z}^{k}, \boldsymbol{\lambda}^{k}, \boldsymbol{\mu}^{k}, \boldsymbol{\nu}^{k}$ have been attained; next, we show how the problems that appear in iteration $k+1$ can be solved.

Step 1 of ADMM consists of solving the problem over $\mathbf{x}$. The problem to be solved is decomposable for each intersection; for the $i$ th intersection the problem is $\mathbf{x}_{i}^{k+1}=$ $\arg \min \mathcal{L}\left(\mathbf{x}_{i}, \mathbf{z}^{k}, \boldsymbol{\lambda}^{k}, \boldsymbol{\mu}^{k}, \boldsymbol{\nu}^{k}\right)$ which can be defined as:

$$
\begin{aligned}
\min & (2 / \rho) \mathbf{f}_{i}^{T} \mathbf{x}_{i}+\left\|\mathbf{x}_{i}-\mathbf{z}_{i}^{k}+\boldsymbol{\nu}_{i}^{k}\right\|_{2}^{2} \\
+ & \sum_{c \in \mathcal{B}_{i}^{O}} \sum_{t \in \mathcal{T}}\left(y_{c, t}+s_{c, t}+W z_{c+1, t}^{n, k}-W N+\lambda_{c, t}^{k}\right)^{2} \\
+ & \sum_{c \in \mathcal{B}_{i}^{I}} \sum_{t \in \mathcal{T}}\left(n_{c, t+1}-n_{c, t}+y_{c, t}-z_{c-1, t}^{y, k}+\mu_{c, t}^{k}\right)^{2}
\end{aligned}
$$

s.t. Constraints (6b), (6c) and (6d),

Formulation (8) is a quadratic program (QP) which can be solved with standard QP solvers. The only limitation in (8) is that the values of constants $z_{c+1, t}^{n, k}, c \in \mathcal{B}_{i}^{O}$ and $z_{c-1, t}^{y, k}$, $c \in \mathcal{B}_{i}^{I}, t \in \mathcal{T}$ are not locally available; however, these quantities can easily be communicated to $I C_{i}$ as they belong to immediate neighboring intersections.

Step 2 of ADMM consists of solving the problem over $\mathbf{z}$. The problem to be solved is $\mathbf{z}^{k+1}=$ $\arg \min \mathcal{L}\left(\mathbf{x}^{k+1}, \mathbf{z}, \boldsymbol{\lambda}^{k}, \boldsymbol{\mu}^{k}, \boldsymbol{\nu}^{k}\right)$ which can be decomposed similarly to step 1 of $\mathrm{ADMM}$, so as $I C_{i}, i \in \mathcal{R}$ has to solve the following unconstrained QP:

$$
\begin{aligned}
\min & \left\|\mathbf{x}_{i}^{k+1}-\mathbf{z}_{i}+\boldsymbol{\nu}_{i}^{k}\right\|_{2}^{2} \\
& +\sum_{c \in \mathcal{B}_{i}^{I}} \sum_{t \in \mathcal{T}}\left(y_{c-1, t}^{k+1}+s_{c-1, t}^{k+1}+W z_{c, t}^{n}-W N+\lambda_{c-1, t}^{k}\right)^{2} \\
& +\sum_{c \in \mathcal{B}_{i}^{O}} \sum_{t \in \mathcal{T}}\left(n_{c+1, t+1}^{k+1}-n_{c+1, t}^{k+1}+y_{c+1, t}^{k+1}-z_{c, t}^{y}+\mu_{c+1, t}^{k}\right)^{2}
\end{aligned}
$$

Formulation (9) is separable for each $z$ variable, hence the solution for the above problem can be obtained in closedform by solving one-dimensional problems of the form $\min _{z}\left\{a z^{2}+b z\right\}$, whose solution is $z=-b /(2 a)$. Similar to step 1 , the only limitation is that $y_{c-1, t}^{k+1}, s_{c-1, t}^{k+1}, \lambda_{c-1, t}^{k}, c \in$ 
$\mathcal{B}_{i}^{I}$ and $y_{c+1, t}^{k+1}, n_{c+1, t}^{k+1}, \mu_{c+1, t}^{k}, c \in \mathcal{B}_{i}^{O}, t \in \mathcal{T}$, are not known to $I C_{i}$ and need to be communicated to $I C_{i}$ from adjacent intersections. Hence, assuming that these quantities are known to appropriate intersections, $\mathbf{z}^{k+1}, i \in \mathcal{R}$ can be computed analytically by solving $N_{I}$ problems in parallel, one at each intersection.

Step 3 of ADMM consists of simply updating the dual variables associated with the coupled equality constraints. $I C_{i}$ needs to perform the following updates, for $t \in \mathcal{T}$ :

$$
\begin{aligned}
& \lambda_{c, t}^{k+1}=y_{c, t}^{k+1}+s_{c, t}^{k+1}+W z_{c, k+1}^{n, k+1}-W N+\lambda_{c, t}^{k}, c \in \mathcal{B}_{i}^{O}, \\
& \mu_{c, t}^{k+1}=n_{c, t+1}^{k+1}-n_{c, t}^{k+1}+y_{c, t}^{k+1}-z_{c-1, t}^{y, k+1}+\mu_{c, t}^{k}, c \in \mathcal{B}_{i}^{I}, \\
& \boldsymbol{\nu}_{i}^{k+1}=\mathbf{x}_{i}^{k+1}-\mathbf{z}_{i}^{k+1}+\boldsymbol{\nu}_{i}^{k} .
\end{aligned}
$$

The computation of updates (12) are based only on local information, while updates (10) and (11) require knowledge of terms $z_{c+1, t}^{n, k+1}, c \in \mathcal{B}_{i}^{O}$ and $z_{c-1, t}^{y, k+1}, c \in \mathcal{B}_{i}^{I} t \in \mathcal{T}$ which can be obtained from neighboring intersections.

From the above analysis, it is clear that each ADMM iteration requires three computation and local communication steps. Communication step 1 can be eliminated as values $z_{c+1, t}^{n, k}, c \in \mathcal{B}_{i}^{O}$ and $z_{c-1, t}^{y, k}, \quad c \in \mathcal{B}_{i}^{I}, t \in \mathcal{T}$ needed in the $k$ th iteration have already been obtained from communication step 3 of the previous iteration. In addition, communication step 3 can be avoided if $I C_{i}$ explicitly computes the $z$-values it needs for computation step 3. In our case, computing the necessary $z$-values of boundary cells of neighboring intersections carries very little computational cost compared to the overall cost of computation steps 1 and 2 , so it is beneficial to trade-off one communication step with some extra computation. To summarize, each iteration of ADMM requires three computation steps and one communication step after computation step 1 .

\section{Phase 2: Distributed rounding}

Having obtained a distributed solution to $\mathcal{R} \mathcal{N} \mathcal{T S C}$ via ADMM, the next step is to round the main decision variables ( $\left.w_{i, t}, i \in \mathcal{R}, t=1, \ldots, T_{w}\right)$ so as to obtain a close to optimal solution to $\mathcal{N} \mathcal{T S C}$. It is important to note that rounding should be performed in a distributed manner in accordance to the variables that are known to each IC, and also that the decision variables need to be rounded-off only for the time-window $T_{w}$ and not for the whole period $T_{o}$.

Towards this direction, we have developed the distributed cumulative departure rate rounding (DCDRR) method, originally proposed as a heuristic for rounding solutions in a centralized setting [5]. The main idea of DCDRR is to attempt to match the cumulative departure rate (CDR) resulting from $\mathcal{R} \mathcal{N} \mathcal{S} \mathcal{C}$, with the CDR obtained from binary decisions for each movement of an intersection. The CDR of phase $p$ of intersection $i$ at time $\tau$ is defined as:

$$
C_{p, \tau, i}=\sum_{t=1}^{\tau} \sum_{c \in \mathcal{D}_{p, i}} y_{c, t}=C_{p, \tau-1, i}+\sum_{c \in \mathcal{D}_{p, i}} y_{c, \tau}
$$

where $\mathcal{D}_{p, i}$ denotes the set of cells immediately downstream all movements of phase $p$ of intersection $i$.

DCDRR is outlined in Algorithm 1. Note that DCDRR requires two rounds of communication between adjacent

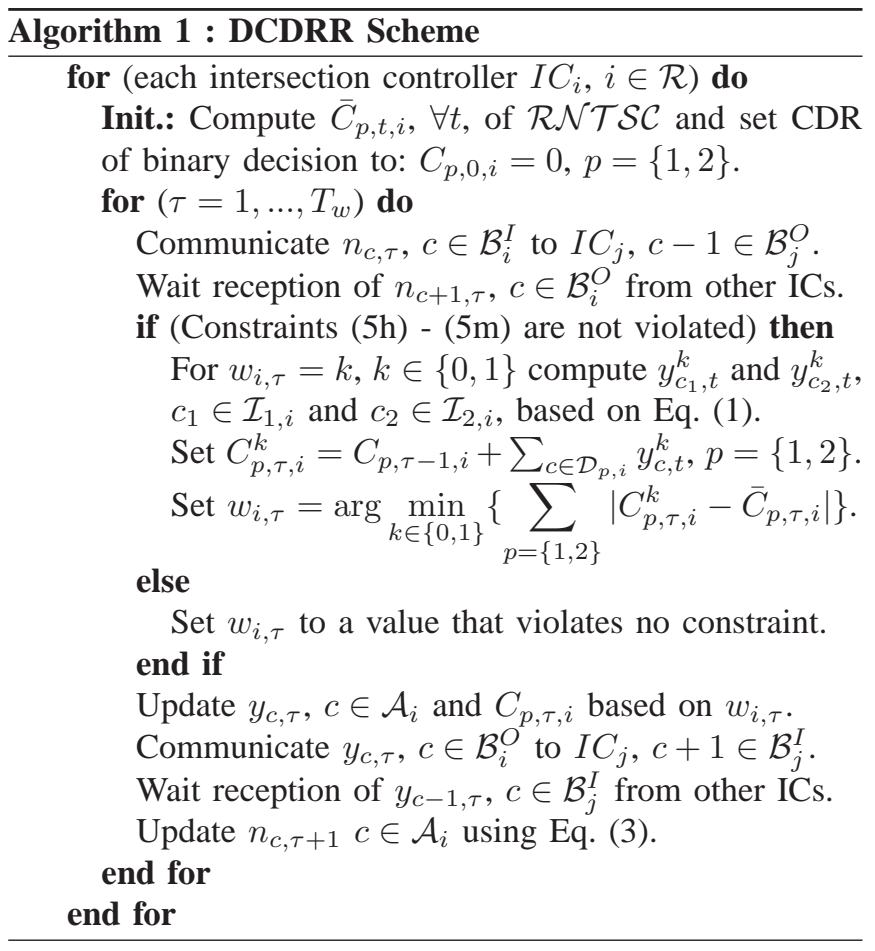

intersections for each time unit which are necessary for the distributed computation of variables $y_{c, t}$ and $n_{c, t}$.

\section{Simulation Results}

The effectiveness of the proposed approach was evaluated for a four-intersection base topology of two-way, two-lane alternating direction arterials as shown in Fig. 1. The freeflow speed and time-step are set to $50 \mathrm{~km} / \mathrm{h}$ and $5 \mathrm{~s}$, respectively, resulting in $70 \mathrm{~m}$-long cells. The distance between intersections is $280 \mathrm{~m}$ ( 4 cells). The capacity of all cells is $\mathrm{Q}=5$ veh./time unit, the jam density is $\mathrm{N}=20$ veh./cell, and $W=0.75$. The maximum and minimum green time are assumed to be equal to $15 \mathrm{~s}$ and $45 \mathrm{~s}$ respectively, i.e. $G_{\min }=3$ and $G_{\max }=8$. The time horizon considered is $T_{h}=1800$ time units $(2.5 h)$, with traffic being generated for 720 time units $(1 h)$. The time horizon is temporally decomposed into 10min intervals $\left(T_{w}=T_{f}=120\right)$. In the evaluation, we consider five randomly generated traffic scenaria corresponding to $10 \%, 30 \%, 50 \%, 70 \%$ and $90 \%$ congestion level. In the ADMM algorithm, we set $\rho=N_{I}$, while for faster convergence we consider an over-relaxation parameter $a=1.6$ (see [11]). Experiments were conducted on a 64-bit Windows 7 desktop computer with Intel i5-3470 processor $(3.2 \mathrm{GHz})$ and $8 \mathrm{~GB}$ of DDR3 RAM; optimization problems were solved using Gurobi [14].

Fig. 2 illustrates the convergence properties of the distributed ADMM algorithm for the 30\% congestion-level traffic scenario when optimizing over one time-window. The figure illustrates that both the primal and dual residuals, which are measures of constraint feasibility and solution optimality [11], respectively, converge quickly with several orders of magnitude improvement in 200 iterations. Fast convergence (in about 50 iterations) is also indicated from the second subfigure, which depicts the total travel time (TTT) 

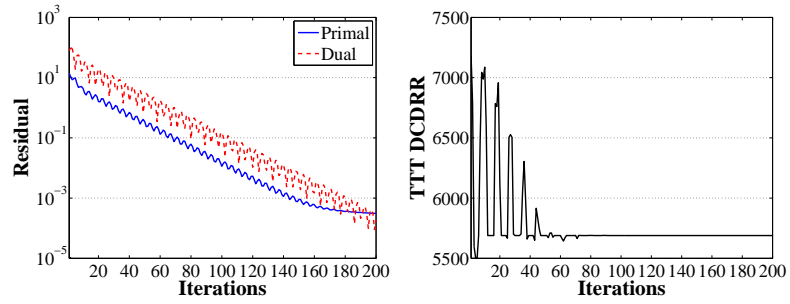

Fig. 2. Convergence properties of the proposed ADMM method.

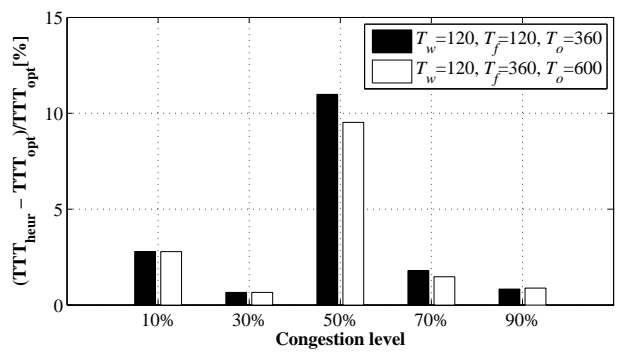

Fig. 3. Comparison between the optimal solution of NTSC and the solution of DCDRR heuristic for a $2 \times 2$ intersection topology.

that would have been obtained from the DCDRR method if the ADMM algorithm was stopped at that iteration. Similar behavior has been observed in other traffic scenarios, with all scenarios examined converging in less than 50 iterations. Hence, we have adopted a stopping criterion of 80 iterations for all considered problem instances.

Fig. 3 depicts the relative deviation of the proposed DCDRR heuristic from the optimal TTT obtained via a MILP solver for the base topology when considering two scenarios with different $T_{f}$ and $T_{o}$ values. Two important observations can be made. First, DCDRR performs very well for all cases considered with the worst performance being within $10 \%$ of the optimal for the $50 \%$ congestion case scenario, while for low and high congestion its performance is very close to optimality (within 2.5\%). Second, larger $T_{f}$ and $T_{o}$ periods have little impact on the overall performance, implying that large look-ahead horizons provide minor benefits.

The scalability properties of the proposed distributed ADMM algorithm is also examined for the base topology under the moderate traffic scenario in Figs. 4(a) and 4(b). Because iterations must be performed synchronously for all intersections, each data point in the figures represents the maximum execution time of any intersection for a specific iteration, while the dotted lines show the nonlinear leastsquares fit of the polynomial model $a_{1} x^{a_{2}}+a_{3}$. It can be observed that the variation of the execution time for specific scenarios is quite small, while the iteration execution time scales almost linearly both to the number of cells $\left(a_{2}=1.06\right)$ and the optimization time $\left(a_{2}=1.19\right)$. This result implies that the proposed approach scales well when expanding the single intersection subproblems in both space and time, and also maintains the online nature of the proposed technique.

\section{CONCLUSIONS \& FUtURE WORK}

In this paper, we have developed a distributed online solution strategy for the network traffic signal control problem

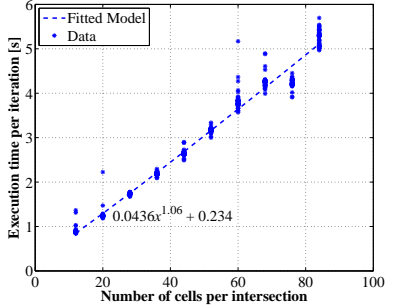

(a)

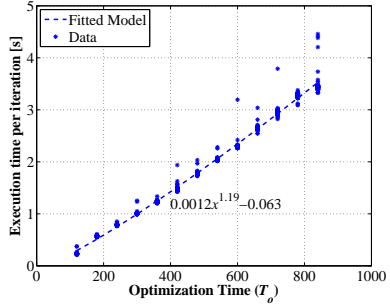

(b)
Fig. 4. Maximum execution time per iteration for all intersections with varying: (a) number of cells per intersection, (b) optimization time.

yielding good optimality, convergence and scaling properties for large problems. To achieve this, the relaxed problem is distributedly solved by an ADMM algorithm after appropriate reformulation and decomposition of the problem in both space and time. Then, the obtained solution is exploited to attain traffic signal plans through distributed rounding. Extending the developed method to different traffic models and transportation problems will be examined in future work.

\section{REFERENCES}

[1] S. G. Shelby, "Single-intersection evaluation of real-time adaptive traffic signal control algorithms," Transportation Research Record, vol. 1867 , no. 1 , pp. 183-192, 2004.

[2] N. H. Gartner, S. F. Assman, F. Lasaga, and D. L. Hou, "A multiband approach to arterial traffic signal optimization," Transportation Research Part B: Methodological, vol. 25, no. 1, pp. 55 - 74, 1991.

[3] D. I. Robertson, "TRANSYT: a traffic network study tool," tech. rep., Transportation Research Laboratory, 1969

[4] W.-H. Lin and C. Wang, "An enhanced 0-1 mixed-integer LP formulation for traffic signal control," IEEE Transactions on Intelligent Transportation Systems, vol. 5, no. 4, pp. 238-245, 2004.

[5] Q. He, W.-H. Lin, H. Liu, and K. Head, "Heuristic algorithms to solve 0-1 mixed integer LP formulations for traffic signal control problems," in IEEE International Conference on Service Operations and Logistics and Informatics (SOLI), 2010, pp. 118-124, IEEE, 2010.

[6] D. Robertson and R. Bretherton, "Optimizing networks of traffic signals in real time-the SCOOT method," IEEE Transactions on Vehicular Technology, vol. 40, pp. 11-15, Feb 1991.

[7] P. Mirchandani and L. Head, "A real-time traffic signal control system: architecture, algorithms, and analysis," Transportation Research Part C: Emerging Technologies, vol. 9, no. 6, pp. 415-432, 2001.

[8] A. G. Sims and K. Dobinson, "The Sydney coordinated adaptive traffic (SCAT) system philosophy and benefits," IEEE Transactions on Vehicular Technology, vol. 29, no. 2, pp. 130-137, 1980.

[9] S. El-Tantawy, B. Abdulhai, and H. Abdelgawad, "Multiagent Reinforcement Learning for Integrated Network of Adaptive Traffic Signal Controllers (MARLIN-ATSC): Methodology and Large-Scale Application on Downtown Toronto," IEEE Transactions on Intelligent Transportation Systems, vol. 14, pp. 1140-1150, Sept 2013.

[10] T. Wongpiromsarn, T. Uthaicharoenpong, Y. Wang, E. Frazzoli, and D. Wang, "Distributed traffic signal control for maximum network throughput," in 15th IEEE International Conference on Intelligent Transportation Systems (ITSC), 2012, pp. 588-595, IEEE, 2012.

[11] S. Boyd, N. Parikh, E. Chu, B. Peleato, and J. Eckstein, "Distributed optimization and statistical learning via the alternating direction method of multipliers," Foundations and Trends in Machine Learning, vol. 3, no. 1, pp. 1-122, 2011.

[12] C. F. Daganzo, "The cell transmission model: A dynamic representation of highway traffic consistent with the hydrodynamic theory," Transportation Research Part B: Methodological, vol. 28, no. 4, pp. 269-287, 1994.

[13] S. Timotheou, C. G. Panayiotou, and M. M. Polycarpou, "Towards Distributed Online Cooperative Traffic Signal Control using the Cell Transmission Model," in Proc. of the 16th IEEE Conf. on Intelligent Transportation Systems, Netherlands, pp. 1737-1742, 2013.

[14] I. Gurobi Optimization, "Gurobi optimizer reference manual," 2013. 